\title{
Editorial
}

\author{
Eric S. Holmboe and Steven J. Durning
}

\section{Understanding the social in diagnosis and error: a family of theories known as situativity to better inform diagnosis and error}

https://doi.org/10.1515/dx-2020-0080

Despite decades of research in understanding and improving clinical reasoning, diagnostic error continues to be a pernicious and pervasive problem globally [1]. One reason for this state of affairs may be the failure to consider influences that are outside the physician's head, yet still envelop the individual clinician during the often complex clinical reasoning process. The traditional view posits that establishing a diagnosis is all in a physician's head; what happens outside of the physician's head is largely noise (e.g., the environment, others in the encounter, artifacts such as the electronic health records [EHRs]). This special edition explores a family of social cognitive theories commonly referred to as situativity theory and proposes a very different way to approach and improve diagnosis, including how it is taught, how it is assessed, and how it should be innovated and researched.

Situativity theory is a group of related theories that are typically placed under the umbrella of social cognitive theory. In short, social cognitive theories attend to the "dynamic and reciprocal interaction of the person, environment, and behavior," where social refers to people and society $[2,3]$. The situativity lens argues that clinical reasoning (the steps up to and including establishing a diagnosis and management plan) emerges from the interactions of the clinician with others in the encounter (e.g., patient, patient's family members, learners, nursing staff, other health care professionals) as well as the environment itself (EHR, point of care resources, number of rooms, etc.). This lens represents a very different view of clinical reasoning and error as a dynamic social (i.e., between people and society) and situated (dependent upon the specific

Corresponding author: Eric Holmboe, MD, Accreditation Council for Graduate Medical Education, 401 North Michigan Avenue, Chicago, IL, 60611, USA, E-mail: eholmboe@acgme.org

Steven J. Durning: Uniformed Services University of the Health Sciences, Bethesda, MD, USA circumstances or context of the encounter) process. Individually different, but related theories in situativity provide unique insights and opportunities for improving our understanding of diagnosis and mitigating error. Indeed, we see diagnostic error as an extreme "phenotype" of clinical reasoning (i.e., clinical reasoning gone awry).

Take, for example, the phenomenon of context specificity, which differs from case (also known as content) specificity. Case specificity argues that physician performance varies based upon the "facts" of the patient's presentation. A clinician may be very good at diagnosing pneumonia but not very good at diagnosing pulmonary embolism or congestive heart failure. In other words, content is important. Context specificity refers to the phenomenon of seeing two patients with the same presenting symptoms and findings (and having the same diagnosis or "content") and yet the clinician comes to two different diagnostic decisions. Why does this happen? What might produce different outcomes for the "same diagnosis," a clear source of unwanted variation in patient care? Content and context specificity might be viewed as two interdependent poles - both are important but overemphasizing one pole over the other creates increased risk for diagnostic error [4].

Situativity, by explicitly incorporating context, provides a unique lens into what is happening in the specific clinical situation, dividing these elements into factors such as physician factors (e.g., sleepiness, burnout), patient factors (e.g., acuity of the presentation, demographic characteristics) and environmental factors (e.g., time for appointment, functionality of EHRs, availability of clinical decisions support). These factors are believed to interact and impact what outcome (e.g., of clinical reasoning) ultimately emerges. For example, using a social lens rebalances the emphasis between the individual clinician and the specifics of the event and enables a deeper exploration of context specificity in particular and diagnostic error more generally.

Four theories that comprise situativity are highlighted in this special issue: situated cognition; distributed cognition; embodied cognition; and ecological psychology 
[5-8]. This family of four theories that comprise situativity share several characteristics that have been coined the "3 E's":

- The embodied mind (the mind interacts with the body; take for example performing a physical examination of the abdomen and the physician's maneuvers that are required for successfully detecting an enlarged spleen);

- The embedded mind (the mind is embedded in the environment; how we use an ultrasound to help detect a pleural effusion); and

- The extended mind (the mind interacts with the larger universe-other people and artifacts in the environment; rounding with an ICU team) [5-8].

These four theories build upon each other. Embodied cognition logically emphasizes the first $\mathrm{E}$ (embodied mind) while ecological psychology stresses the importance of interactions between individuals in a clinical encounter and these individuals' interactions with their environment. This includes affordances (i.e., properties of objects in the environment influencing actions) and effectivities (i.e., qualities of the environment that bring about an effect). Situated cognition provides a framework that incorporates both embodied cognition and ecological psychology tenets, viewing the encounter as an interaction between physician, patient and environmental factors. Finally, distributed cognition allows for the use of the situated cognition approach in groups of individuals as well as providing a framework for looking at clinical reasoning and error over time (e.g., patient presents in emergency room and then is seen on the wards followed by the ICU) $[9,10]$.

This family of related social cognitive theories (situativity) also shares an important relationship with sociotechnical theory (STS), a theory that explores the relationships between people and technology [11]. A brief description of STS will help the reader of this special edition understand how STS helps to orient situativity into systems thinking. STS is grounded in two key principles. The first principle acknowledges that the interaction of social and technical factors plays a major role in system performance. STS explicitly recognizes that these relationships within systems can be complex and unpredictable, especially the "socio" aspect, as humans are not machines. Clinical reasoning is embedded within systems; if the social and technical aspects of the system are poorly designed and integrated, poor system performance is likely to follow. By extension, one can reasonably hypothesize a negative, and at times unpredictable, impact on diagnosis $[12,13]$. The second STS principle argues that optimization of technical or social, to the exclusion of the other, increases the probability of unpredictable and harmful effects on system performance.

One group of papers in this edition explores context specificity through an important lens - the role and impact of distracting contextual factors that are part of everyday life in clinical settings (i.e., microsystems). For example, Konopasky and colleagues, using a controlled experimental design that introduced distracting contextual factors systematically with case videos, found significant differences in an outpatient unstable angina case but no differences in a stable diabetes case [13]. This study provides interesting insights for future "in vivo" studies of context [13]. Soh and colleagues found that the presence of distracting contextual factors can distort the sequence of clinical reasoning tasks and clinical reasoning accuracy in experienced physicians [14]. In a second study, Konopasky and colleagues found linguistic differences in the thinking of experienced physicians in the setting of distracting contextual factors, shedding light on the value of using a social cognitive theoretical framework (situativity) when exploring clinical reasoning and error [15]. Ramini and colleagues further found more expressions of uncertainty in the presence of distracting contextual factors [16]. In summary, distractions can be viewed as contextual factors that degrade performance and reduce optimization.

In the context of healthcare delivery and core activities like clinical reasoning, situativity and STS helps us focus on the interdependent experience of humans with both the social and technical aspects of the whole healthcare system. In STS parlance, this is known as "joint optimization." The late systems engineer Donella Meadows argued if you really want to understand a system, then the best method is to observe its actual performance [17]. The value of innovative techniques such as observation, linguistic analysis, ethnography, phenomenology, and other qualitative methods are highlighted in this special edition as they allow for the investigator to explore dynamic social emergent phenomena such as clinical reasoning and error $[15$, $18,19]$. Situativity and STS can help guide those observations, but observation is an essential pathway to understanding the impact of context on clinical reasoning performance in complex clinical settings. Several papers use different qualitative approaches, from unannounced standardized patients to ethnography and the ethical considerations involved in observation that help add to our understanding of the role observation can serve in advancing clinical reasoning $[18,20,21]$.

Finally, this family of four situativity theories embraces the important social and technical aspects of the healthcare workplace as well as the tenets of relational 
autonomy and optimization. We would argue that situativity further provides a portable framework that can be readily applied to the healthcare system to understand both success and failure in clinical reasoning (physician, patient, environmental factors). Situativity is derived from different scientific roots than STS and can be leveraged to understand diagnosis and error with the concepts of the 3 E's. These "macro" lenses help us understand why error occurs and points to potential steps that can be taken to mitigate error. Situativity serves as a useful lens to enable this "joint optimization" of clinical reasoning between patient, healthcare professionals, and technology within an overall system. This situativity lens also does not negate the importance of understanding the "in the head" clinical reasoning processes, but rather begins to help us understand the other "external" factors affecting clinical reasoning performance. It is fair to hypothesize that the lack of attention to the social aspects of clinical reasoning may in part explain the sobering level of diagnostic error still present in current clinical practice.

Rounding out this special edition are a compilation of provocative essays and useful review articles pertaining to situativity that will help deepen the reader's understanding of the more focused papers highlighted earlier. Rencic and colleagues conducted a literature review through the lens of situativity and discuss clinical reasoning performance through this synthesis [12, 22]. Schuwirth and colleagues review the assessment of decision making through different perspectives to include situativity [23]. Olson and colleagues stress the need to move from largely parallel to integrated, collaborative clinical reasoning between and among teams to include research and practice implications [24]. Readers are also encouraged to review the graphic descriptions of each of the four related theories that comprise situativity in this special edition as well as the paper by Torre et al. outlining teaching and assessment implications with using a situativity perspective [5-10].

The persistent, continual data on the scale of harm from diagnostic error mandates we examine our own approaches to studying, assessing, and improving clinical reasoning [1]. The ultimate purpose of health professions education and research it to improve the lives of patients, families, and communities. The substantial and harmful impact of diagnostic error requires us to challenge our own assumptions and existing paradigms. Considering what may improve diagnostic reasoning and mitigate error beyond what is going on in the individual clinician's head could help to dramatically advance our field. To do so requires new theoretical approaches (e.g., situativity), different methodological approaches (e.g., those that capture nonlinearity through observation and innovative qualitative methods) and new study designs that allow for the understanding of factors beyond a clinician's knowledge and skill that impacts clinical reasoning performance (e.g., exploring context specificity).

We hope you find that this special edition provides a unique perspective into diagnosis and error by providing a more holistic, systems view of clinical reasoning that can shape how we provide instruction, perform assessment, and conduct research this field.

Research funding: None declared.

Author contributions: All authors have accepted responsi bility for the entire content of this manuscript and approved its submission.

Competing interests: Authors state no conflict of interest.

\section{References}

1. Balogh E, Miller B, Ball J. Improving diagnosis in health care. Washington, DCs: National Academy of Medicine; 2015.

2. LaMorte WW. Behavioral change models. The social cognitive theory. Boston University; 2019. Available from: http://sphweb. bumc.bu.edu/otlt/MPH-Modules/SB/

BehavioralChangeTheories/BehavioralChangeTheories5.html [Accessed 30 April 2020].

3. Bandura A. Social cognitive theory. In: Vasta R, editors. Annals of child development. Six theories of child development. Greenwich, CT: JAI Press; 1989, Vol. 6. 1-60 pp.

4. Govaerts MJB, van der Vleuten CPM, Holmboe ES. Managing tensions in assessment: moving beyond either-or thinking. Med Educ 2019;53:64-75.

5. Daniel M, Durning S, Wilson E, Abdoler E, Torre D. Situated cognition: clinical reasoning and error are context dependent. Diagnosis (Berl) 2020;7:341-2.

6. Wilson E, Seifert C, Durning S, Torre D, Daniel M. Distributed cognition; interactions between individuals and artifacts. Diagnosis (Berl) 2020;7:343-4.

7. Daniel M, Wilson E, Torre D, Durning S, Lang V. Embodied cognition; knowing in the head is not enough. Diagnosis (Berl) 2020;7:337-8.

8. Daniel M, Torre D, Durning S, Wilson E, Rencic J. Ecological psychology; diagnosis and treating patients in complex environments. Diagnosis (Berl) 2020;7:339-40.

9. Merkebu J, Battisone M, McMains K, McOwen K, Witkop C, Konopasky A, et al.. Situativity: a family of social cognitives theories for understanding clinical reasoning and diagnostic error. Diagnosis (Berl) 2020;7:169-76.

10. Torre D, Durning S, Rencic J, Lang V, Holmboe E, Daniel M. Widening the lens on teaching and assessing clinical reasoning: from "in the head" to "out in the world" . Diagnosis (Berl) 2020;7: $181-90$. 
11. Walker GH, Stanton NA, Salmon PM, Jenkins DP. A review of sociotechnical systems theory: a classic concept for new command and control paradigms. Theor Issues Ergonomics Sci 2008;9:479-499.

12. Rencic J, Schuwirth L, Gruppen L, Durning S. A situated cognition model for clinical reasoning performance assessment: a narrative review. Diagnosis (Berl) 2020;7:227-40.

13. Konopasky A, Artino A, Battista, A, Ohmer M, Hemmer P, Torre D, et al. Understanding context specificity: the effect of contextual factors on clinical reasoning. Diagnosis (Berl) 2020;7:257-64.

14. Soh M, Konopasky A, Durning S, Ramani D, McBee E, Radcliffe T, et al. Sequence matters: patterns in task-based clinical reasoning. Diagnosis (Berl) 2020;7:281-9.

15. Konopasky A, Durning S, Artino A, Ramani D, Battista A. The linguistic effects of context specificity: exploring affect, cognitive processing, and agency in physicians' think-aloud reflections. Diagnosis (Berl) 2020;7:273-80.

16. Ramini D, Soh M, Merkebu J, Durning S, Battista A, McBee A, et al. Examining the patterns of uncertainty across clinical reasoning tasks: effects of contextual factors on clinical reasoning process. Diagnosis (Berl) 2020;7:299-305.

17. Meadows DH. Thinking in systems: a primer. White River Junction, VT: Chelsea Green Publishing; 2008.
18. Chopra V. Focused ethnography: a new tool to study diagnostic errors?. Diagnosis (Berl) 2020;7:211-4.

19. Briedis C, Briediene R. Phenomenological analysis of diagnostic radiology: description and relevance to diagnostic errors. Diagnosis (Berl) 2020;7:215-25.

20. Berdahl C, Schriger DL. Study design and ethical considerations related to using direct observation to evaluate physician behavior; reflections after a recent study. Diagnosis (Berl) 2020; 7:205-9.

21. Schwartz A, Peskin S, Spiro A, Weiner S. Direct observation of depression screening: identifying diagnostic error and improving accuracy through unannounced standardized patients. Diagnosis (Berl) 2020;7:251-6.

22. Rencic J, Schuwirth L, Gruppen L, Durning S. Clinical reasoning performance assessment: using situated cognition theory as a conceptual framework. Diagnosis (Berl) 2020;7: 241-9.

23. Schuwirth L, Durning S, King S. Assessment of clinical reasoning: three evolutions of thought. Diagnosis (Berl) 2020;7:191-6.

24. Olson A, Durning S, Fernandez C, Sick B, Lane K, Rencic J. Teamwork in clinical reasoning - cooperative or parallel play? Diagnosis (Berl) 2020;7:307-12. 